\title{
Distributed Collector System: Modelling, Control and Optimal Performance
}

\author{
A. Alsharkawi, J.A. Rossiter \\ Department of Automatic Control and Systems Engineering \\ University of Sheffield \\ Mappin St., Sheffield, S1 3JD, UK \\ e-mail: aalsharkawi1@ sheffield.ac.uk, j.a.rossiter@sheffield.ac.uk
}

\begin{abstract}
Continual increases in electricity demand, the global rise in oil consumption and prices, the contribution of oil consumption to greenhouse gases emissions and the fact that the supply of fossil fuels will eventually run out are all driving factors in the need for renewable energy solutions. This paper gives an overview of the main concentrated solar thermal power technologies with an emphasis on the modelling and control of conventional parabolic trough technology. Specific focus is given to the benefits of model-based predictive control in a distributed solar collector field of a parabolic trough plant.
\end{abstract}

\section{Key words}

CSP technologies; Parabolic trough plant; Model-based predictive control; Hierarchical control structure

\section{Introduction}

In 1972 the US National Science Foundation stated that "Solar energy is an essentially inexhaustible source potentially capable of meeting a significant portion of the nation's future energy needs with a minimum of adverse environmental consequences ... The indications are that solar energy is the most promising of the unconventional energy sources". In fact all forms of existing energy are solar in origin. Solar energy is converted into electrical energy by two main approaches; a direct approach using photovoltaic (PV) technology and an indirect approach using concentrated solar power (CSP) technology, where the electricity is produced by thermal means [1]. In the long-term CSP technology will represent the most reliable energy source with a large installed capacity and thus a key role in grid stabilisation and power security, while the application of PV technology will be limited to decentralised and remote applications [2].

CSP plants generate electricity by converting the solar energy into stored heat energy. The heat energy is then used to drive a power cycle, for instance a steam turbine or a heat engine [2], [3]. Yet, CSP implementation is faced with the drawbacks of high investment cost and the intermittency of solar energy [4]. Technological developments targeting the main elements of a CSP plant and large-scale power production are the only way to overcome these drawbacks [4], [3]. Advances in CSP technologies can be found in [5]. Mass power production can be achieved by either having a hybrid operation that combines a CSP plant with a conventional fossil fuel power plant or by having a CSP plant backed up with an efficient heat storage system. Both solutions will allow a compensation for any short time fluctuations in the solar energy and increase the annual operating hours [4].

From the control point of view, one of the challenging issues in a CSP plant is to maintain the thermal process variables close to their desired levels. In contrast to conventional power plant where fuel is used as the manipulated variable, in a CSP plant, solar energy cannot be manipulated. In fact, solar energy acts as a disturbance due to its change on a daily and seasonal basis. The development of efficient control techniques able to cope with this issue will benefit in longer operating hours and electricity cost reductions [6].

Parabolic trough technology is one of the CSP technologies that has received a great deal of attention in terms of modelling and control and indeed a special interest in applying Model-based Predictive Control (MPC) techniques to address the earlier mentioned control problem is also evident. However, the reasons behind the interest in this type of technology is not clearly stated and nor is the motivation to utilize such an advanced control technique. Hence, this paper aims to show the potential benefits of parabolic trough technology compared with the other CSP technologies and moreover to highlight the benefits of applying MPC techniques. The paper also refers to some of the key and recent work in modelling and control of parabolic trough plants and points out where future research is likely to be focused.

The paper is organized as follows: The next section gives an overview of the main CSP technologies from the aspects of basic concepts, advantages, disadvantages and applications. This is then followed by two sections briefly presenting the modelling and control of parabolic trough plants. Finally, a section is devoted to opportunities in the control of solar energy.

\section{CSP Technologies}

CSP technologies have four main elements in common; a concentrator, a receiver, a heat transfer fluid and a power conversion [2]. Some researchers tend to classify CSP technologies according to the concentrator sun tracking mechanism into a single and two axis tracking technologies [5], while others prefer to classify them according to the distribution of the focused solar 
radiation on an observer into line and point focus technologies [7]. The category of the single axis tracking technologies or the line focus technologies mainly comprises the parabolic trough and linear Fresnel reflector technologies. The category of the two axis tracking technologies or the point focus technologies mainly comprises the central receiver and parabolic dish technologies [5], [2].

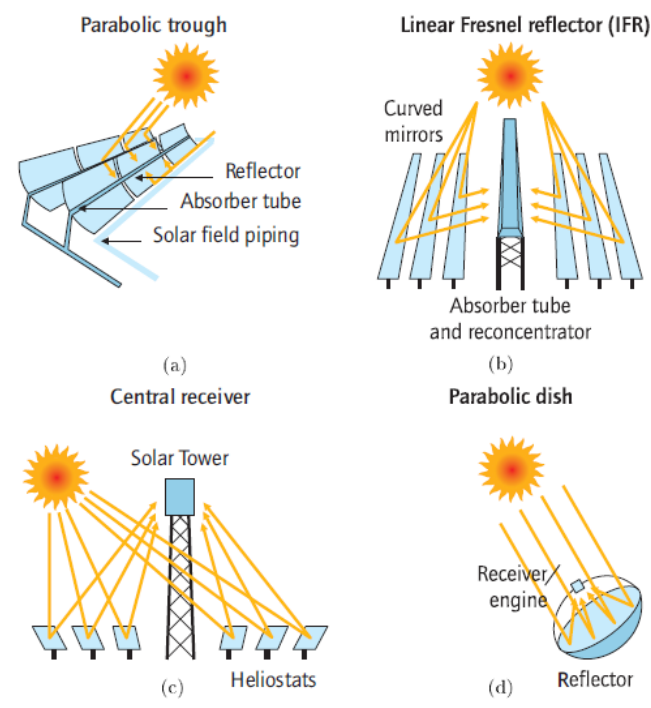

Fig. 1. CSP Technologies [8].

\section{A. Parabolic Trough Technology}

Concentrators of this technology are sheets of reflective material which are parabolic in shape. Incident solar radiation is concentrated by the parabolic concentrator onto a receiver tube placed at its focal line (Fig. 1a). Because the parabolic trough collector can only make use of direct solar radiation it is provided with a single axis tracking mechanism [9]. The collector can either track the sun from north to south or from east to west [10].

The receiver tube contains thermal oil that circulates through the solar field and is heated to a temperature of approximately $400^{\circ} \mathrm{C}$. The heated oil passes through a series of heat exchangers to produce steam that is used to drive a conventional steam turbine to generate electricity [2]. Direct Steam Generation (DSG) technology can also be used by having water in the receiver tube [9].

1) Advantages: i) Reliable and mature technology with years of operating experience [4], [2]; ii) Concept of hybrid operation has been commercially proven [4], [2]; iii) Modular and scalable which allows a largescale power production [4], [2]; iv) Storage systems capability [2]; v) Compared with central receiver and parabolic dish technologies it has shown an efficient land usage and required less materials [2].

2) Disadvantages: i) High investments costs [5]; ii) Operating temperature is limited to a certain level [2]; iii) Requirements of a stable support structure [4].

3) Applications: Parabolic trough technology is best suited for centralized power production [7], [2]. The US grid-connected Solar Electricity Generating Systems (SEGS) power plants in California represent the most successful parabolic trough plants with a total installed capacity of $354 \mathrm{MW}$ [9].

\section{B. Linear Fresnel Reflector Technology}

This is an attempt to enhance and simplify the traditional parabolic trough technology by flattening or nearly flattening the parabolic trough reflectors into a set of rows capable of tracking the sun about one axis and concentrate the solar radiation on to a fixed downward facing receiver parallel to the reflector's rotational axis (Fig. 1b) [10], [5]. DSG is well suited for this type of technology [8], [11].

1) Advantages: i) Requires less support structure as reflectors positioned close to the ground [10], [11];

ii) Having a stationary receiver eliminates the need for ball joints [2], [12]; iii) The flat reflectors are less expensive compared to parabolic trough reflectors [2], [11]; iv) Requires less land usage [12], [8]; v) Reflectors are easier to clean [12].

2) Disadvantages: i) Lower thermal performance is the price of the lower investments and operation and maintenance costs [8], [11]; ii) Incorporating a storage capacity is challenging [8], [11]; iii) More complex tracking mechanism [13].

3) Applications: Similar to parabolic trough technology, linear Fresnel technology is suited for centralized power production. One of the recent implementations of this technology is the gridconnected Puerto Errado 2 in Spain with a total installed capacity of $30 \mathrm{MW}$ [11].

\section{Central Receiver Technology}

A large number of heliostats (reflectors) grouped together with a two axis sun tracking mechanism for each one of them. Reflectors are used to concentrate the solar radiation onto a central receiver placed on top of a tower (Fig. 1c). Solar energy is absorbed at the central receiver by a Heat Transfer Fluid (HTF) to be used in a conventional power cycle [10].

1) Advantages: i) Able to reach an operating temperature over $1000^{\circ} \mathrm{C}$ [2]; ii) Capability of hybrid operation [4], [2]; iii) Modular and scalable which allows a large-scale power production [4]; iv) High storage temperatures [2].

2) Disadvantages: i) Requirements of a stable support structure [4], ii) Long-term commercial performance still need to be proven [11].

3) Applications: Appropriate technology for centralized power production as discussed in [7], [2]. The commercial PS10 in Spain demonstrates a gridconnected central receiver solar power plant with a total installed capacity in the range of $10 \mathrm{MW}$ [14].

\section{Parabolic Dish Technology}

Concentrator of a parabolic dish technology is dishshaped reflector that focuses the incident solar radiation at its focal point where a receiver is positioned (Fig. 1d). HTF running through the receiver is heated up and used by a heat engine for electricity production [10].

1) Advantages: i) Exhibits the highest energy conversion efficiency [8], [11]; ii) Can achieve temperatures beyond $1500^{\circ} \mathrm{C}[10]$; iii) Capability of hybrid operation [4], [2]; iv) Modular and scalable which allows a large-scale power production [2], [10]; v) Some operational experience gained from 
research projects and prototypes [4], [2]; vi) Cooling systems for the exhaust heat are not required [11].

2) Disadvantages: i) Commercial performance and operation is still yet to be proven [2]; ii) Concept of hybrid operation is not proven yet [4]; iii) Benefits of large-scale power production still need to be proven [2]; iv) High investment cost due to the requirements for a solid and reliable support structure and the dual axis tracking mechanism [4], [11].

3) Applications: Parabolic dish technology is believed to be suitable for distributed power production as a stand-alone units in remote areas and small communities [7], [11]. Technology implementation is restricted to prototypes operated successfully over the past decade with installed capacities in the range of 10-100 kW [11]. The Boeing SES dish is a US prototype which uses Stirling cycle motors and has delivered over 10,000 hr of operation [5].

\section{E. Discussion}

Although over the past years CSP plants showed a rapid growth in the global market, it is not yet competitive economically with conventional power plants [11]. Labour and land cost, incorporation of a storage system, plant size [8], [11], technologies used [8] and plant maturity [11] have a significant impact on the investment and electricity generating costs for any CSP plant [8], [11]. Despite the enhancements that could be done to achieve a reduction in investment and electricity generating costs, parabolic trough technology is commercially considered to be the most economic and reliable technology available [2]. Over 90\% of the current installed CSP capacity is accounted for by parabolic trough plants [11].

In a parabolic trough plant, a highly skilled and trained operator with a very good knowledge of the sun's daily and seasonal path, observations of changing weather and years of experience is responsible for maintaining the outlet fluid temperature at a desired level regardless of any changes in the sun intensity, the collector inlet temperature and the ambient temperature, by adjusting the flow rate of the HTF circulating through the collectors within given upper and lower limits. However, the limited performance of a human controller implies the importance of developing effective automatic control [15]. Automatic control plays a crucial role in the improvement of the efficiency, performance and associated running costs of a parabolic trough plant [16].

As parabolic trough technology represents the most wide spread CSP technology and due to the high influence of automatic control on the overall plant performance, it is not surprising that the literature is rich with work devoted to modelling and control of parabolic trough plants. The next two sections discuss briefly the modelling and control approaches of parabolic trough plants.

\section{Modelling Approaches}

Models can be classified into three main categories; theoretical models, empirical models and semi-empirical models [17].

\section{A. Theoretical Models}

Theoretical models are developed based on first principles and describe the physical behaviour of a process [17]
Since the early attempts to control the temperature of the HTF in a parabolic trough plant, the energy balance relations for the receiver tube in eqn. (1) and the fluid in eqn. (2) describing the collector dynamics, have established a fundamental role of developing models used in the design of numerous control techniques [6]. Both lumped and distributed parameter models can be obtained from eqns. $(1,2)$ [18].

$$
\begin{gathered}
\rho_{m} C_{m} A_{m} \frac{\partial T_{m}}{\partial t}=n_{\circ} I G-H_{l} G\left(T_{m}-T_{a}\right) D H_{t}\left(T_{m}-T_{f}\right) \\
\rho_{f} C_{f} A_{f} \frac{\partial T_{f}}{\partial t}+\rho_{f} C_{f} q \frac{\partial T_{f}}{\partial x}=D H_{t}\left(T_{m}-T_{f}\right)
\end{gathered}
$$

The subindex $m$ refers to the receiver tube metal and $f$ to the fluid, $\rho$ : density $\left(\mathrm{kg} / \mathrm{m}^{3}\right), C$ : specific heat $\left(\mathrm{J} / \mathrm{kg}^{\circ} \mathrm{C}\right), A$ : cross-sectional area $\left(\mathrm{m}^{2}\right), T$ : temperature $\left({ }^{\circ} \mathrm{C}\right), n_{\circ}$ : optical efficiency, $I$ : solar irradiance $\left(W / m^{2}\right), G$ : optical aperture $(m), H_{l}$ : global coefficient of thermal losses $\left(W / \mathrm{m}^{2 \circ} \mathrm{C}\right)$, $T_{a}$ : ambient temperature $\left({ }^{\circ} \mathrm{C}\right), D$ : inner diameter of the receiver tube $(m), \quad H_{t}$ : coefficient of metal-fluid transmission $\left(\mathrm{W} / \mathrm{m}^{2}{ }^{\circ} \mathrm{C}\right), q$ : oil flow $\left(\mathrm{m}^{3} / \mathrm{s}\right), x$ : length $(\mathrm{m})$.

\section{B. Empirical Models}

Empirical models are obtained by the use of experimental data related to specific operating conditions [17]. The collector dynamics have been modelled empirically by observing a step response in an open-loop fashion. The response can be approximated by a simple first order system, as shown in eqn. (3), with a time delay relatively small compared to the system time constant.

$$
g\left(z^{-1}\right)=z^{-k} \frac{b z^{-1}}{1-a z^{-1}}
$$

Eqn. (3) is still an approximated model and not adequate enough to capture an important dynamic phenomena of the plant known as anti-resonant modes [6]. The phenomena are described in [19] as resonance characteristics of the collector dynamics that lie within the desired control bandwidth. Failure to accurately model these resonance characteristics will result in a poor oscillatory performance and low stability margins. Hence, a nonlinear model or several high order linear models for different operating points are required [20]. In [21], for instance, the free response of a plant is modelled by a nonlinear version of the AutoRegressive with eXogeneous inputs (ARX) model by the application of neural identification using a static (non-recurrent) neural network and the forced response of the plant is modelled by linear Controlled AutoRegressive Integrated Moving Average (CARIMA) models obtained from the Pseudo Random Binary Sequence (PRBS) identification technique.

\section{Semi-empirical Models}

Semi-empirical models are a combination of theoretical and empirical models in such a way that experimental data is used to calculate the numerical value(s) of the physical parameter(s) in a theoretical model [17]. The trade-off between model simplicity and the ability to describe the dynamics of a plant sufficiently motivated the author in [22] to develop two slightly different nonlinear models from the basic physical relations. The models are linear in the parameters, thus can be easily 
estimated on-line and compensate for any time-varying effects or modelling errors. Under the assumption that the system is composed of three main parts: the supply tube, the receiver tube (heated part) and the return tube, a nonlinear grey-box model based on first principles and tuned using real experimental data is presented in [18].

Semi-empirical models receive more interest in the process industry. Although theoretical models provide a physical insight into the process and cover a wide range of operation, their development is quite expensive and time consuming. In addition, some model parameters are not easily obtained. Empirical models are still easier to develop than theoretical models, however, they cover only a limited range of operation. Semi-empirical models on the other hand incorporate conceptual understanding, cover a wider range of operation than empirical models and require less effort to develop than theoretical models [17].

\section{Control Approaches}

Adjusting the flow rate of the HTF in a distributed collector field in order to maintain a desired outlet fluid temperature will result in a significant variations in the collector dynamics (e.g. the response rate and the time delay) which in turn will make the job of a controller with fixed parameters a real challenge [23], [24]. Tuning a fixed (proportional-integral-derivative) PID controller with low gain will lead to a poor performance and a tightly tuned controller might lead to high oscillations [6]. Furthermore, such a system imposes constraints on the fluid flow rate, outlet fluid temperature and the difference between outlet and inlet fluid temperatures for safety and energy efficiency [25]. Such issues necessitate the use of more advanced control techniques. The next sub-section presents an overview of the state-of-the-art in controlling the outlet fluid temperature in parabolic trough plants.

\section{A. State-of-the-Art}

Numerous control techniques have been proposed in the literature to address the control challenges of the outlet fluid temperature in a parabolic trough plant. Some of these control techniques are in the form of: i) an adaptive (proportional-integral) PI controller based on a pole assignment approach [26]; ii) a robust PI controller with reset action on its integral term [27]; iii) a PID controller complemented with a filter to counteract the resonance dynamics effects [28]; iv) a nonlinear PID controller with time varying gain [29]; v) a robust PID controller with fixed parameters based on the quantitative feedback theory (QFT) [30]; vi) a feedback linearization [31]; vii) an adaptive nonlinear control using feedback exact linearization together with a lyapunov's approach [32]; viii) an indirect adaptive nonlinear control based on a recurrent neural network and the output regulation theory [33]; ix) an internal model control [34], and x) a fuzzy logic control [35]. A feedforward term is a fundamental element in most of these control frameworks in order to mitigate the effect of the measured disturbances on the plant dynamics. Different forms of MPC have been also proposed by many researchers [36]. MPC and its implementation to a parabolic trough plant is presented in the next sub-section in more detail.

It should be pointed out that the aim of this sub-section is not to compare the different proposed control techniques, but rather to provide references to some of the key and recent work and give a general idea of some of the various types that have been proposed. The performance of each of these control techniques was validated with different design assumptions and at different operating conditions so inappropriate for a fair comparison.

\section{B. Model-based Predictive Control}

The design concept underpinning MPC is to imitate human behaviour. In a particular situation and based on past information and internal model, a set of control actions are selected and expected to lead to the best predicted outcome over a limited horizon. The planned control actions/strategy are updated continually as more information becomes available. Thus the main components of a predictive control law can be summarized by the following [37]:

- Output predictions based on a process model.

- Some performance measure to define the optimal future control actions.

- Receding horizon: control actions are updated and modified at every sampling instant.

Applying MPC to address the outlet oil temperature control problems in a parabolic trough plant can be beneficial for several reasons; time delays are implicitly considered due to the predictive nature of MPC; the predicted behaviour gives the chance to avoid any undesired dynamics by selecting the appropriate set of control actions; the system constraints are handled online in a systematic fashion and the feedforward term is taken into account automatically [37], [36]. Most of the proposed MPC algorithms can be found in the adaptive, robust, gain scheduling and nonlinear form [6].

1) Adaptive MPC: The idea of adaptive control is to tune the controller parameters on-line in a process where the dynamics change frequently in an unpredictable manner. This can be approached by describing the control law in terms of the on-line estimated process model parameters [17]. One of the early applications of adaptive MPC to a solar power plant is presented in [38]. The adaptive MPC is developed based on a simple linear model of the process and the resulting control law is linear and can be described by a few parameters. In order to obtain an approximation of the true controller parameters, a set of Ziegler-Nichols-type functions were considered to relate the control law parameters to the process model parameters. More recently, a constrained nonlinear adaptive model-based predictive control based on an affine state-space three layered neural network was developed [39]. A dual unscented Kalman filter is considered for the on-line recursive updating of the neural network weights and state estimation.

2) Robust MPC: In contrast to adaptive control, a robust control scheme can cope with changes to process model parameters using a suitable constant gain feedback controller as long as the parameter changes are within certain bounds [40]. A robust MPC based on a simple linear model of a plant with bounded errors is proposed in [41]. Model parameters were allowed to vary within a certain range in order to cope with the changing dynamics 
and the parameters uncertainty level is determined by a robust identification technique. A hybrid approach that combines the strengths of MPC and sliding mode control (SMC) is presented in [42]. The resulting controllers are believed to present a high degree of robustness when they are appropriately tuned. Lately, robustness of stability against parameters uncertainty and measurement errors in a nonlinear MPC has been taken care of by simply including a candidate Lyapunov function in the objective function and the constraints of the controller [43].

3) Gain Scheduling MPC: The performance requirements in a gain scheduling approach can be met by designing several feedback controllers with constant gains that correspond to a number of operating points. Hence, the implementation requires a look-up table to store the values of the controller gains and a criteria to relate the changes in a process dynamics to the appropriate controller gain [40]. High order CARIMA type models obtained from inputoutput data of a plant were used for different operating points in a gain scheduling MPC approach [20]. As the plant dynamics are mainly affected by the changes in the fluid flow, two tables of the process and the controller parameters were obtained for different fluid flow values. An alternative gain scheduling MPC approach, but also based on the fluid flow value, is proposed in [44] where linear ARMAX models were identified on-line for different operating points and used for an indirect adaptive MPC controller.

4) Nonlinear MPC: Linear control techniques can be effective in physical processes, which exhibit nonlinear behaviour to a small degree, for example where one is limited to a narrow range of operation. Otherwise, traditional linear control techniques may not be adequate and nonlinear control techniques can be an option to enable performance improvements [17]. For an MPC control scheme presented in [21], the response of a plant is divided into a forced and free terms. A linear model is used for the forced response to obtain a set of control actions, while a nonlinear model of the free response is used to handle the effect of the disturbances. A recent application of a nonlinear MPC is presented in [43]. A distributed parameter model is used for the simulated process and a lumped parameter model with time delay is used for prediction. The main contribution of this work is that the parameters of the prediction model do not require any identification or adaptation in order to meet the expected results, which implies a reduction in the computational cost when computing the control algorithm.

Once again, this section has demonstrated a large body of research focussed on applying differing forms of MPC, but as yet a useful and insightful comparison seems to be lacking.

\section{Opportunities}

Reduction in investment and operating costs and an increase in solar plant performance can make solar energy more economical [13]. Advanced control techniques can reduce operating costs and increase plant performance [45]. However, most of the control techniques focus on a certain level of automatic control and neglect other levels of process automation, which results in a poor performance at some operating points, particularly during the start up and shut down of the plant. During the start up, the plant is controlled in manual mode by the plant operator until conditions to change to automatic control mode are reached, which is inefficient and time consuming [16]. In order to extend the automation of the process to other levels and improve the final plant performance, hierarchical control approaches are proposed in [25], [16], [45].

The idea of a hierarchical control structure was first presented in [25] to optimize the electricity production process in solar power plants with distributed collectors. The use of a multilayer hierarchical control structure is coming from the fact that the problem involves systems with different dynamical behaviour and time scales. The generic control structure is composed of the following four main layers:

1) The regulation layer is concerned with typical set point temperature tracking and disturbance rejection where simply any control technique can be used.

2) The set point optimization layer is concerned with obtaining the most adequate set points considering the actual operating conditions and plant constraints.

3) The daily optimization layer is concerned with the determination of the daily operating hours of the plant.

4) The weekly optimization layer is concerned with the operational scheduling of the plant within a weekly planning period.

Applying a hierarchical control approach to a solar power plant can benefit in maximizing the electricity production, extend the lifetime of the various elements of the plant, reduce the risk of controller saturation and limit the tasks of the plant operator. In [16], a two-layer hierarchical control strategy is described. The upper layer is implemented using two different approaches for set point optimization in the steady state and the lower layer is a combination of a simple feedforward and feedback controllers for reference tracking and disturbance rejection. The hierarchical structure is extended in [45] to include a third layer for operational scheduling and the set point optimization layer is computed taking into account the dynamic behaviour of the plant while the regulation layer is controlled by an adaptive PI controller.

\section{Conclusion and Future Directions}

The features of the main CSP technologies have been presented and it has been demonstrated that parabolic trough technology is widely accepted and has shown excellent performance in the commercial power industry. Moreover, due to the important part of automatic control in the overall plant performance, some of the key and recent efforts in modelling and control of parabolic trough plants are also presented. Notably conclusions and avenues for future study are:

- For accurate modelling of the plant the dynamic phenomena of anti-resonant modes must be taken care of but as yet there is no convergence in the literature on whether nonlinear models or 
gain scheduling of high order linear models are to be preferred.

- There is some consensus that semi-empirical models are preferable in general.

- Many control techniques have been used, but an effective comparison seems to be lacking.

- There has been substantial interest in the benefits of applying MPC but as yet a reliable comparison and consensus is lacking.

- The adoption of hierarchical control structures is likely to be the future of controlling parabolic trough plants which moreover allow for effects such as weather prediction and variation in electricity demands.

\section{References}

[1] DY Goswami, F Kreith, and JF Kreider. Principles of solar engineering. CRC Press, 2000.

[2] $\mathrm{R}$ Aringhoff, European Solar Thermal Power Industry Association, IEA Solar PACES Implementing Agreement, et al. Concentrated Solar Thermal Power-Now! Greenpeace, 2005.

[3] C Márquez Salazar. An overview of csp in europe, north africa and the middle east. CSP today, London, 2008.

[4] F Trieb, O Langnib, and H Klaib. Solar electricity generation-a comparative view of technologies, costs and environmental impact. Solar Energy, 1997.

[5] D Mills. Advances in solar thermal electricity technology. Solar Energy, 2004.

[6] EF Camacho, M Berenguel, FR Rubio, and D Martínez. Control of solar energy systems. Springer, 2012.

[7] H Klaiß, R Köhne, J Nitsch, and U Sprengel. Solar thermal power plants for solar countries-technology, economics and market potential. Applied energy, 1995.

[8] C Philibert. Technology Roadmap: Concentrating Solar Power. OECD/IEA, 2010.

[9] A Fernández-García, E Zarza, La Valenzuela, and M Pérez. Parabolic trough solar collectors and their applications. Renewable and Sustainable Energy Reviews, 2010.

[10] SA Kalogirou. Solar thermal collectors and applications. Progress in energy and combustion science, 2004.

[11] Concentrating Solar Power: Technology Brief. IEA-ETSAP, IRENA, 2013.

[12] A Giostri, M Binotti, P Silva, E Macchi, and G Manzolini. Comparison of two linear collectors in solar thermal plants: Parabolic trough vs fresnel. In ASME 2011 5th International Conference on Energy Sustainability, 2011.

[13] EF Camacho, T Samad, M Garcia-Sanz, and I Hiskens. Control for renewable energy and smart grids. The Impact of Control Technology, Control Systems Society, 2011.

[14] M Medrano, A Gil, I Martorell, X Potau, and LF Cabeza. State of the art on high-temperature thermal energy storage for power generation. part 2-case studies. Renewable and Sustainable Energy Reviews, 2010.

[15] T Stuetzle, N Blair, JW Mitchell, and WA Beckman. Automatic control of a 30 mwe segs vi parabolic trough plant. Solar energy, 2004

[16] CM Cirre, M Berenguel, L Valenzuela, and R Klempous. Reference governor optimization and control of a distributed solar collector field. European Journal of Operational Research, 2009.

[17] DE Seborg, TF Edgar, and DA Mellichamp. Process dynamics and control. Hoboken, NJ: John Wiley \& Sons; 2nd ed., 2004.

[18] M Gálvez-Carrillo, R De Keyser, and C Ionescu. Nonlinear predictive control with dead-time compensator: Application to a solar power plant. Solar energy, 2009.

[19] A Meaburn and FM Hughes. Resonance characteristics of distributed solar collector fields. Solar Energy, 1993.

[20] EF Camacho, M Berenguel, and FR Rubio. Application of a gain scheduling generalized predictive controller to a solar power plant. Control Engineering Practice, 1994.
[21] MR Arahal, M Berenguel, and EF Camacho. Neural identification applied to predictive control of a solar plant. Control Engineering Practice, 1998.

[22] R Pickhardt. Nonlinear modelling and adaptive predictive control of a solar power plant. Control Engineering Practice, 2000.

[23] R Pickhardt and R Neves da Silva. Application of a nonlinear predictive controller to a solar power plant. In Control Applications, 1998. Proceedings of the 1998 IEEE International Conference on. IEEE, 1998.

[24] EF Camacho and M Berenguel. Application of generalized predictive control to a solar power plant. 1994.

[25] M Berenguel, CM Cirre, R Klempous, H Maciejewski, M Nikodem, J Nikodem, I Rudas, and L Valenzuela. Hierarchical control of a distributed solar collector field. In Computer Aided Systems Theory- EUROCAST 2005. Springer, 2005.

[26] EF Camacho, FR Rubio, and FM Hughes. Self-tuning control of a solar power plant with a distributed collector field. Control Systems, IEEE, 1992.

[27] A Vidal, A Banos, JC Moreno, and M Berenguel. Pi+ ci compensation with variable reset: application on solar collector fields. In Industrial Electronics, 2008. IECON 2008. 34th Annual Conference of IEEE. IEEE, 2008.

[28] JD Alvarez, JE Normey-Rico, and M Berenguel. Design of pid controller with filter for distributed parameter systems. In Advances in PID Control, 2012.

[29] R Neves-Silva. Non linear control of a distributed solar field. In Renewable Energy Research and Applications (ICRERA), 2013 International Conference on. IEEE, 2013.

[30] CM Cirre, JC Moreno, M Berenguel, and JL Guzmán. Robust control of solar plants with distributed collectors. In Proc. of the 2010 IFAC Int. Symp. on Dynamics and Control of Process Systems, DYCOPS, 2010.

[31] CM Cirre, M Berenguel, L Valenzuela, and EF Camacho. Feedback linearization control for a distributed solar collector field. Control Engineering Practice, 2007.

[32] M Barao, JM Lemos, and RN Silva. Reduced complexity adaptive nonlinear control of a distributed collector solar field. Journal of process control, 2002.

[33] J Henriques, P Gil, A Cardoso, P Carvalho, and A Dourado. Adaptive neural output regulation control of a solar power plant. Control Engineering Practice, 2010.

[34] JD Álvarez, Ramon Costa-Castelló, M Berenguel, and LJ Yebra. A repetitive control scheme for distributed solar collector field. International Journal of Control, 2010.

[35] FR Rubio, M Berenguel, and EF Camacho. Fuzzy logic control of a solar power plant. Fuzzy Systems, IEEE, 1995.

[36] EF Camacho and C Bordons. Model predictive control. Springer, 2004.

[37] JA Rossiter. Model-based predictive control: a practical approach. CRC press, 2003.

[38] EF Camacho, $\mathrm{M}$ Berenguel, and $\mathrm{C}$ Bordons. Adaptive generalized predictive control of a distributed collector field. Control Systems Technology, IEEE Transactions on, 1994.

[39] P Gil, J Henriques, A Cardoso, P Carvalho, and A Dourado. Affine neural network-based predictive control applied to a distributed solar collector field. 2014

[40] PA Ioannou and J Sun. Robust adaptive control. Courier Dover Publications, 2012.

[41] EF Camacho and M Berenguel. Robust adaptive model predictive control of a solar plant with bounded uncertainties. International journal of adaptive control and signal processing, 1997.

[42] M Perez de la Parte, CM Cirre, EF Camacho, and M Berenguel.Application of predictive sliding mode controllers to a solar plant. Control Systems Technology, IEEE, 2008.

[43] GA Andrade, DJ Pagano, JD Álvarez, and M Berenguel. A practical nmpc with robustness of stability applied to distributed solar power plants. Solar Energy, 2013.

[44] R Pickhardt. Application of adaptive controllers to a solar power plant using a multi-model description. In American Control Conference, 1998. Proceedings of the 1998. IEEE, 1998.

[45] EF Camacho and AJ Gallego. Optimal operation in solar trough plants: A case study. Solar Energy, 2013. 